\section{PREDICTION OF NOSOCOMIAL INFECTIONS AFTER PEDIATRIC CARDIAC SURGERY}

\author{
S.O. Algra ${ }^{1,2}$, M.M.P. Driessen ${ }^{2}$,
} A.W.L. Schadenberg' ${ }^{2}$, A.N.J. Schouten ${ }^{2}$, F. Haas ${ }^{1}$, C.W. Bollen², N.J.G. Jansen²

${ }^{1}$ Department of Pediatric Cardiothoracic Surgery, ${ }^{2}$ Department of Pediatric Intensive Care, University Medical Center Utrecht, Utrecht, The Netherlands

Background and aims: Nosocomial infections are a common complication after cardiac surgery in children. Identifying patients at risk for this complication is essential to minimize postoperative morbidity and mortality. This study describes the incidence and characteristics of nosocomial infections after pediatric cardiac surgery at our institution. Furthermore, predictors for these infections are evaluated for their prognostic value.

Methods: Aretrospectivecohortstudywasperformed including all pediatric patients who underwent cardiac surgery with use of cardiopulmonary bypass from April 2006 until May 2009. Nosocomial infections were defined using Center of Disease Control-criteria. Clinically available variables, known at 48 hours postoperatively, were included in a univariate analysis to evaluate their association with nosocomial infections. A multivariable analysis was performed to identify independent risk factors and the prognostic value of the model was assessed.

Results: Following 416 procedures, nosocomial infections occurred in 102 patients (24.7\%). Four independent risk factors were identified by multivariable analysis: lower weight, open sternum postoperatively, presence of a noncardiac comorbidity and postoperative stay at the intensive care $>48$ hours. A prognostic model combining these factors yielded a predictive value of $78.2 \%$.

Conclusions: At 48 hours after pediatric cardiac surgery, four independent variables predict the occurrence of an infection: lower weight, presence of a noncardiac comorbidity, open sternum postoperatively and current admission at the intensive care. However, when combined in a model, the prognostic value of these predictors is poor. Consequently, early recognition of patients at high risk for infection remains difficult.

\section{A COMPARISON OF EMPIRIC ANTIBIOTICS AND SENSITIVITIES OF ORGANISMS CAUSING ACQUIRED NEONATAL SEPTICAEMIA}

\author{
G. Inglis ${ }^{1,2}$, A. Stewart ${ }^{3}$ \\ ${ }^{1}$ Neonatology, Royal Brisbane and Women's \\ Hospital, ${ }^{2}$ Department of Child Health, The \\ University of Queensland, Brisbane, QLD, \\ Australia, ${ }^{3}$ Neonatology, Royal Hospital for Sick \\ Children, Glasgow, UK
}

Aims: To compare empiric antibiotics with the sensitivities of organisms causing acquired septicaemia in infants in a tertiary neonatal unit

Methods: We performed a retrospective audit of culture-positive septicaemia in the Grantley Stable Neonatal Unit between January 2005 and December 2009. Cases were identified using the unit's database. Relevant data were extracted from the database or, where necessary, by reviewing patient files.

Results: During the study period there were 7572 admissions, with 120 episodes of septicaemia in 109 patients. The most common organisms isolated were enteric Gram-negative bacilli $(n=34)$, coagulasenegative Staphylococcus (CONS) species $(n=31)$ and Staphylococcus aureus $(n=24)$. Sufficient data for analysis were available for 116 episodes in 105 patients. Empiric antibiotics prescribed most frequently were ampicillin, cefotaxime, gentamicin and metronidazole. Number of antibiotics used empirically in each episode ranged from 2 to 4 . The most commonly used antibiotic regimen was ampicillin and cefotaxime. The chosen regimen included antibiotic/s that matched the reported sensitivities in 56/116 (48\%) episodes. Of the 60 cases where the reported sensitivities did not include the empiric antibiotics, the regimen nevertheless provided partial cover in 23 . In 19 of the remaining 37 cases, the infecting organism was CONS

Conclusions: Empiric antibiotic regimens provided adequate or partial cover against septicaemiacausing organisms in two-thirds of cases. In a significant minority the regimen was inadequate. However, half of these were caused by CONS, which is known to be less virulent than other septicaemia-causing organisms. Broader empiric antibiotic cover may be impractical but knowledge of prior colonising organisms may help. 